\title{
Differentiation of Entamoeba histolytica from Entamoeba dispar by nested multiplex polymerase chain reaction
}

\section{Original Article}

\author{
Faten A. Mohammed, Afaf A. Taha, Marwa A. Salama
}

Department of Medical Parasitology, Faculty of Medicine, Zagazig University, Zagazig, Egypt

\begin{abstract}
Background: Amoebiasis is a parasitic disease caused by the intestinal protozoon Entamoeba histolytica. Microscopic examination fails to differentiate E. histolytica from the morphologically identical nonpathogenic Entamoeba dispar. To avoid unnecessary treatment of individuals infected with nonpathogenic $E$. dispar, it is essential to differentially diagnose infections caused by pathogenic from nonpathogenic Entamoeba spp.

Objective: The aim of this study was to assess the efficacy of nested multiplex PCR (NM PCR) technique as a diagnostic method for differentiating infections caused by E. histolytica and E. dispar.

Materials and methods: Stool samples collected from patients with and without symptoms of amoebiasis were screened for E. histolytica/E. dispar trophozoites/cysts by microscopic examination. NM PCR was performed on a total of 52 samples targeting the genus-specific 16S-like ribosomal RNA gene for simultaneous, differential detection of E. histolytica and E. dispar.

Results: NM PCR detected E. histolytica at $439 \mathrm{bp}$ and E. dispar at $174 \mathrm{bp}$, and it was positive in 31 out of 32 cases with a sensitivity of $96.85 \%$. From those, $17(32.7 \%)$ samples were positive for E. histolytica, $12(23.1 \%)$ for E. dispar, and three $(5.7 \%)$ for both species. In addition, NM PCR diagnosed E. dispar in one of the negative controls with a specificity of $95 \%$.
\end{abstract}

Conclusions: NM PCR is useful for the specific detection of E. histolytica and E. dispar in stool samples.

Key Words: Entamoeba dispar, Entamoeba histolytica, nested multiplex polymerase chain reaction.

Received: 10 November 2016, Accepted: 7 January 2017.

Corresponding Author: Faten A. Mohammed, Tel. : +20-10-2031-1001, E-mail: fatenmr@yahoo.com.

ISSN: 1687-7942, Vol. 10, No. 1 \& 2.

\section{INTRODUCTION}

Amoebiasis is a parasitic disease caused by the intestinal protozoon Entamoeba histolytica. It is considered the third cause of death due to parasitic infections in humans after malaria and schistosomiasis, and was reported to be responsible for about 40,000-100,000 deaths/year in Australia $^{[1]}$. It is worldwide in distribution, and is considered a serious health threat in tropical and subtropical developing areas; it is also considered a problem in travelers and immigrants in the developed world ${ }^{[2]}$. The prevalence and severity of E. histolytica infection vary from one geographical area to another and from one case to another, with the highest prevalence found in regions with low hygienic conditions ${ }^{[3,4]}$.

Six of the morphologically identical Entamoeba spp. (E. histolytica, E. dispar, E. moshkovskii, E. polecki, E. coli, and E. hartmanni) can live in the human intestine. Only E. histolytica causes pathological lesions, whereas the others are considered as nonpathogenic ${ }^{[5]}$ while E. moshkovskii is debated. In 1997, the WHO declared E. histolytica as a pathogenic species and E. dispar as a nonpathogenic species ${ }^{[6]}$.
Traditionally, the laboratory diagnosis of Entamoeba spp. is based on their morphology on microscopic examination of stool $^{[7]}$. However, morphological identification fails to differentiate E. histolytica from the identical nonpathogenic E. dispar ${ }^{[8]}$. Stool culture followed by isoenzyme analysis allows the differentiation, but it requires several weeks and needs special laboratory facilities, making it impractical for use in the routine diagnosis of intestinal amoebiasis ${ }^{[8]}$. Isoenzyme analysis for detecting the $\mathrm{N}$-acetyl galactosamine/galactose (Gal/ GalNAc) lectin of E. histolytica or E. dispar has been reported to give excellent sensitivity in endemic areas ${ }^{[9]}$. Stool antigen assays have been reported to be more sensitive and specific than microscopy. The coproantigen enzyme-linked immunosorbent assay (ELISA) technique has been suggested for screening Entamoeba spp. in routine diagnostic procedures and epidemiological studies. Although more reliable and specific than microscopy, it does not differentiate the species ${ }^{[10,1]}$.

Additional methods for species differentiation have been used to avoid unnecessary treatment of individuals infected with the nonpathogenic species ${ }^{[12]}$. PCR demonstrated excellent sensitivity and specificity 
compared with microscopy. It has been endorsed by several studies ${ }^{[9,13]}$ as the method of choice for clinical and epidemiological studies of amoebiasis. Multiplex PCR is a molecular biology technique used for the amplification of multiple targets in a single PCR reaction ${ }^{[14]}$; therefore, it is considered more cost-effective and less laborious than other PCR methods ${ }^{[15]}$.

In the present study, NM PCR was evaluated as a diagnostic method for the simultaneous differential detection of E. histolytica and E. dispar in stool samples.

\section{MATERIALS AND METHODS}

Study design: In this descriptive analytical study, fresh stool samples were collected without preservatives in sterile containers from persons with stool analysis requests attending the Outpatient Clinics of Zagazig University Hospitals during the period between April 2014 and April 2015.

Stool samples were screened microscopically for the presence of $E$. histolytica/E. dispar cysts/trophozoites complex or any other intestinal parasites. According to the results of the stool analysis, a total of 52 stool samples were selected for testing by NM PCR and were classified into the following groups: group 1 (symptomatic group; G1) included 16 E. histolytica/E. dispar-positive samples for cysts/trophozoites from patients showing symptoms of amoebiasis (dysentery, tenesmus, abdominal pain and tenderness, alternating bowel habits, flatulence, nausea, vomiting, or fever); group 2 (asymptomatic group; G2) included 16 E. histolytica/E. dispar-positive samples from asymptomatic patients; and group 3 included 20 samples negative for $E$. histolytica/E. dispar cysts/trophozoites and negative for any other parasitic infections (negativecontrol group; G3). About $2 \mathrm{~g}$ of each sample was aliquoted into $1.5-\mathrm{ml}$ screw-cap tubes and stored at $-20^{\circ} \mathrm{C}$ until DNA extraction and NM PCR targeting the 16S-like rRNA gene.

Stool examination: Naked-eye macroscopic examination of collected samples determined consistency, color, odor, and presence of blood, mucous, or pus. For microscopic examination, all samples were subjected to saline and iodine wet-mounts and formol-ether concentration technique within $2 \mathrm{~h}$ after fecal sample collection as previously described ${ }^{[16,17]}$. The results of the stool analysis were recorded either as positive or as negative for $E$. histolytica/E. dispar, according to detection of cysts or trophozoites of either species.

Genomic DNA extraction: DNA extraction was carried out using Favor Prep stool DNA Isolation Mini Kit (Cat. No. FASTI001; Favorgen Biotech Corporation, Pingtung, Taiwan), according to the manufacturer's guide, with modification. The thermal shock procedure was carried out (cycling of samples by deep-freezing in liquid nitrogen for $5 \mathrm{~min}$, followed by immediate transfer into a $95^{\circ} \mathrm{C}$ water bath for $5 \mathrm{~min}$ (repeated for five cycles), and incubation for $1 \mathrm{~h}$ at $95^{\circ} \mathrm{C}$ and for $10 \mathrm{~min}$ at $56^{\circ} \mathrm{C}$. The purified DNA was measured for concentration and purity and kept at $-20^{\circ} \mathrm{C}$ until further use.

Nested multiplex polymerase chain reaction: A primary PCR was performed targeting the 16S-like rRNA gene for the detection of Entamoeba genus. Used primers (Table 1) included the forward primer EF and the reverse primer ER amplifying the genus-specific gene, which is $\sim 800 \mathrm{bp}$. Subsequently, the primary PCR products were subjected to secondary PCR for Entamoeba spp.-specific characterization. Amplification was achieved using primer sets EHF and EHR to detect the E. histolytica fragment segment at $439 \mathrm{bp}$ and primer sets EDF and EDR to detect the E. dispar fragment segment at $174 \mathrm{bp}$.

The reaction components and the cycling conditions were selected according to Ngui et al. ${ }^{[12]}$ with modification in the form of $12.5 \mu 1$ master mix, $200 \mathrm{nM}$ from each primer, and $3 \mu \mathrm{l}$ of the template DNA for the primary reaction and $1 \mu \mathrm{l}$ for the secondary reaction in a total volume of $25 \mu \mathrm{l}$ and $56^{\circ} \mathrm{C}$ annealing temperature for the primary and $48^{\circ} \mathrm{C}$ for the secondary reactions. The amplified products were visualized after $1.5 \%$ agarose gel electrophoresis with ethidium bromide staining. Control samples without (negative control) and with Entamoeba spp. genomic DNA (positive control) were included in each PCR run. Two standard strains, E. histolytica HM-1:IMSS and E. dispar SAW760, were used as positive controls.

Table 1: Oligonucleotide primers used in NM PCR

\begin{tabular}{lll}
\hline & Primer names & Primer sequence \\
\hline Genus-specific primers (used in the first PCR) & EF (forward primer) & $5^{\prime}$-TAA GAT GCA GAG CGA AA-3' \\
& ER (reverse primer) & $5^{\prime}$-GTA CAA AGG GCA GGG ACG TA-3' \\
$\begin{array}{l}\text { Species-specific primers (used in the second } \\
\text { NM PCR) }\end{array}$ & EHF (forward primer) & $5^{\prime}$-AAG CAT TGT TTC TAG ATC TGA G-3' \\
& EHR (reverse primer) & 5'-AAG AGG TCT AAC CGA AAT TAG-3' \\
& EDF (forward primer) & $5^{\prime}$-TCT AAT TTC GAT TAG AAC TCT-3' \\
& EDR (reverse primer) & $5^{\prime}$-TCC CTA CCTATT AGA CAT AGC-3' \\
\hline
\end{tabular}


Statistical analysis: The results were calculated, tabulated, and statistically analyzed using statistical computer program SPSS, version II, for Windows $7^{[18]}$. A $P$-value of less than 0.05 was considered to be statistically significant.

Ethical consideration: A description of the objectives and methodology of the study was given to the patients or parents/guardians before sample collection, and a written or thumb-printed informed consent was obtained. The study was approved by the Committee of Research, Publications, and Ethics of the Faculty of Medicine, Zagazig University, Egypt.

\section{RESULTS}

The present study was conducted on 52 stool samples to detect and differentiate $E$. histolytica from $E$. dispar infections by NM PCR. E. histolytica was detected at 439 bp and E. dispar at $174 \mathrm{bp}$ (Figure 1). Of 32 microscopypositive samples for Entamoeba spp. (Table 2), 31 samples were successfully amplified and characterized as Entamoeba spp. on the basis of amplicon size with a sensitivity of $96.9 \%$ (Table 3). E. histolytica was detected in $17 / 32(53.12 \%)$ samples, E. dispar in 11/ 32 (34.38\%) samples, and three $(9.37 \%)$ samples were positive for both (Table 2). Examination of 20 microscopy-negative samples confirmed that 19 samples were negative for E. histolytica infection, and one sample was positive for E. dispar (Table 2 ) with $95 \%$ specificity (Table 3 ).

In $\mathrm{G} 1$, of 16 symptomatic cases, $12(75.0 \%)$ samples were positive by NM PCR for E. histolytica, two (12.5\%) samples for E. dispar, one (6.25\%) sample for both, and one $(6.25 \%)$ sample was negative. Of the 16 positive samples from G2 of asymptomatic cases, five (31.2\%) samples were positive by NM PCR for E. histolytica, nine $(56.3 \%)$ samples for E. dispar, and two (12.5\%) samples for both. Of the 20 microscope-negative samples in $\mathrm{G} 3$, one sample was diagnosed as $E$. dispar positive
(Table 4). The detection rate of E. histolytica was higher $(75 \%)$ in symptomatic G1 than in nonsymptomatic G2 $(31.2 \%)$, whereas the detection rate of $E$. dispar was higher $(56.3 \%)$ in $\mathrm{G} 2$ than in G1 (12.5\%), and this relationship was statistically significant $(P<0.05)$ (Table 4$)$.

\section{DISCUSSION}

E. histolytica, the causative agent of human intestinal and extra-intestinal amoebiasis, is a protozoon responsible for significant morbidity and mortality mainly in developing and some of the developed countries ${ }^{[19,20]}$. The diagnosis and epidemiology of amoebiasis have become more complex with the introduction of the Entamoeba

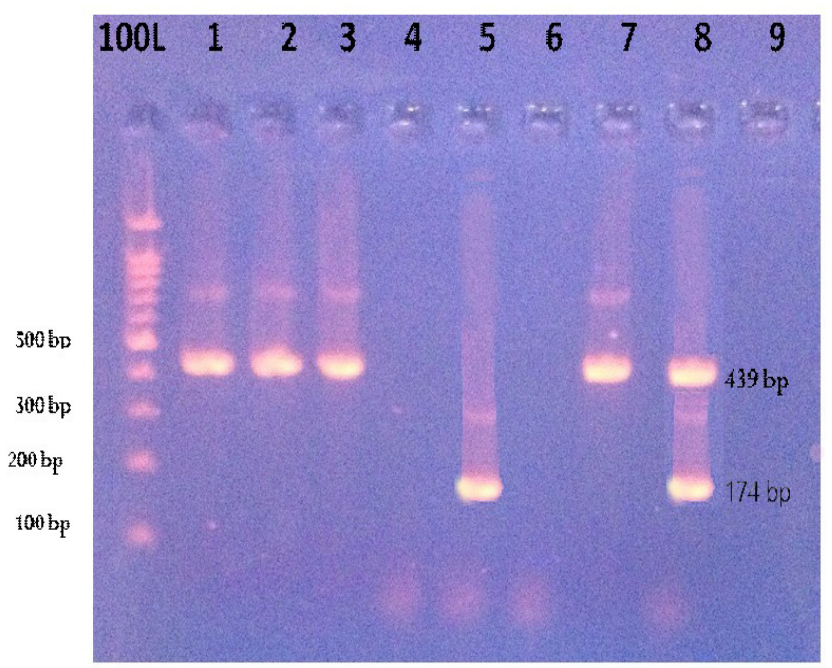

Figure 1: Agarose gel electrophoresis of NM PCR products amplified by E. histolytica-specific primers (EHF and EHR) and $E$. dispar-specific primers (EDF and EDR). Lane 100L: 100 bp DNA marker ladder. E. histolytica PCR products at $439 \mathrm{bp}$. E. dispar PCR product at 174 bp. Lanes 1-3: E. histolytica positive samples. Lanes 4, 6: Negative samples. Lane 5: E. dispar-positive sample. Lane 7: E. histolytica positive control. Lane 8: mixed infection of E. histolyticalE. dispar. Lane 9: Negative control.

Table 2: Differential detection of E. histolytica and E. dispar in stool specimens by NM PCR

\begin{tabular}{|c|c|c|c|c|c|}
\hline \multirow[b]{3}{*}{$\begin{array}{l}\text { Results of } \\
\text { microscopy }\end{array}$} & \multicolumn{5}{|c|}{ Results of NM PCR [n (\%)] } \\
\hline & \multicolumn{4}{|c|}{ Positive } & \multirow{2}{*}{$\frac{\text { Negative }}{\text { Total }}$} \\
\hline & E. histolytica & E. dispar & $\begin{array}{l}\text { E. histolytica } \\
\text { and } E \text {. dispar }\end{array}$ & Total & \\
\hline Positive $(\mathrm{N}=32)$ & $17(53.13)$ & $11(34.38)$ & $3(9.37)$ & $31(96.87)$ & $1(3.12)$ \\
\hline Negative $(\mathrm{N}=\mathbf{2 0})$ & $0(0)$ & $1(5.0)$ & $0(0)$ & $1(5)$ & $19(95)$ \\
\hline Total $(\mathrm{N}=52)$ & $17(32.69)^{\mathrm{a}}$ & $12(23.08)^{\mathrm{a}}$ & $3(5.77)^{\mathrm{a}}$ & $32(61.54)^{\mathrm{a}}$ & $20(38.46)^{\mathrm{a}}$ \\
\hline
\end{tabular}

NM: nested multiplex, a Percentage from total 
Table 3: Sensitivity, specificity, and positive and negative predictive values of NM PCR for Entamoeba spp. complex in relation to microscopic examination

\begin{tabular}{lcc}
\hline Screening tests (PCR) & \multicolumn{2}{c}{ Results } \\
\hline True positives (TP) & 31 & \\
False negative (FN) & 1 & 96.9 \\
False positives (FP) & 19 & 95 \\
True negatives (TN) & & 96.9 \\
Sensitivity [TP/(TP+FN)] (\%) & & 95 \\
Specificity [TN/(TN+FP)] (\%) & & \\
PPV [TP/(TP+FP)] (\%) & & \\
NPV [TN/(TN+FN)] (\%)
\end{tabular}

NPV: negative predictive value, PPV: positive predictive value.

Table 4: Results of NM PCR in the different groups studied

\begin{tabular}{|c|c|c|c|c|c|}
\hline Groups & $\begin{array}{l}\text { E. histolytica } \\
\text { [n }(\%)]\end{array}$ & $\begin{array}{l}\text { E. dispar } \\
{[\mathrm{n}(\%)]}\end{array}$ & Mixed [n (\%)] & Negative [n (\%)] & $\begin{array}{l}\text { Statistical } \\
\text { analysis }\end{array}$ \\
\hline G1 (N=16) & $12(75)$ & $2(12)$ & $1(6.25)$ & $1(6.25)$ & $\begin{array}{l}\chi^{2}=6.055 \\
P=0.014\end{array}$ \\
\hline G2 $(\mathrm{N}=16)$ & $5(31.2)$ & $9(56.3)$ & $2(12.5)$ & $0(0)$ & \\
\hline G3 $(N=20)$ & $0(0)$ & $1(5)$ & $0(0)$ & $19(95.0)$ & \\
\hline Total $(\mathrm{N}=52)$ & $17(32.7)^{\mathrm{a}}$ & $12(23.1)^{\mathrm{a}}$ & $3(5.7)^{\mathrm{a}}$ & $20(38.5)^{\mathrm{a}}$ & \\
\hline
\end{tabular}

G1: symptomatic group, G2: asymptomatic group, G3: noninfected control group, a: Percentage from total.

complex, which included pathogenic E. histolytica and nonpathogenic $E$. dispar ${ }^{[21]}$. For the assessment of the true prevalence of pathogenic E. histolytica and the accurate diagnosis of amoebiasis, it is important to detect and distinguish E. histolytica from nonpathogenic species ${ }^{[8]}$. Microscopic examination of stool samples was the most commonly used diagnostic test, particularly in areas with limited resources; however, it cannot differentiate between these three species ${ }^{[22]}$. Differentiation between the morphologically identical pathogenic and nonpathogenic species can be achieved genetically, biochemically, and immunologically ${ }^{[5]}$, as well as by performing isoenzyme analysis, but the procedure requires to be preceded by stool culture, which is time consuming and laborious with a sensitivity of only about $50 \%{ }^{[8]}$. Even the commercial ELISA-based method for specific identification and detection of E. histolytica in fecal specimens ${ }^{[23]}$ has shown poor sensitivity and specificity in many studies because of cross-contaminations with other parasites ${ }^{[24,25]}$. In the last decade, molecular-based diagnostic tests have gained importance in the diagnosis of many infectious diseases including amoebiasis to overcome the problems of conventional methods with the advantages of increased sensitivity, specificity, and simplicity ${ }^{[26,27]}$. Several PCR assays designed to differentiate $E$. histolytica from E. dispar have been described ${ }^{[15,28,29]}$. Most of them targeted either the small-subunit ribosomal RNA gene or specific episomal repeats species. Accordingly, the present study was conducted to assess the application of NM PCR to recognize and differentiate E. histolytica and E. dispar directly from stool samples.

In the present study, NM PCR diagnosed 31 out of 32 samples collected from symptomatic patients, and failed to diagnose one Entamoeba-positive sample with 96.9\% sensitivity. Similarly, El Sobky et al. ${ }^{[30]}$ reported a sensitivity of NM PCR of $96.4 \%$ compared with trichromestained preparations $(75 \%)$. The negative result by NM PCR in the present study in one microscopy-positive sample could be explained by the presence of DNA from an Entamoeba spp. other than E. dispar/E. histolytica. It was suggested by Ngui et al. ${ }^{[12]}$ and Fallah et al. ${ }^{[31]}$ that the samples detected by microscopy but not PCR may belong to other Entamoeba spp. such as E. coli, E. hartmanni, and E. polecki. The results of the present study are comparable with those of Fallah et al. ${ }^{[31]}$ who recorded positive PCR in 25 out of 31 stool samples diagnosed by microscopic methods. A slightly lower sensitivity (94\%) was reported by Khairnar and Parija ${ }^{[8]}$ whose results showed negative NM PCR in 12 Entamoeba spp.-microscopy-positive stool samples. Failure to detect pathogens in stool samples by PCR may be explained by the complexity of the specimens for direct PCR testing due to the presence of PCR inhibitors such as heme, bilirubin, bile salts, and complex carbohydrates, which are often coextracted along with pathogenic $\mathrm{DNA}^{[9]}$. Therefore, optimization of the fecal 
DNA extraction procedure is critical to the success of PCR. Stool samples positive by microscopy and negative by NM PCR could also contain a low number of parasites, below the PCR detection limit ${ }^{[12]}$.

In their study, Khairnar and Parija ${ }^{[8]}$ reported that NM PCR results were negative for the 35 negative-control stool samples with $100 \%$ specificity. In the present study, the NM PCR result was positive for one sample, which showed negative results by microscopy. This may be explained by possible contamination of the sample by DNA from other sources $^{[31]}$, resulting in a decreased specificity of $95 \%$ (Table 3). In the study by Gachuhi et al. ${ }^{[32]}$, microscopic examination identified $21.3 \%$ of their patients to be infected with $E$. histolytica/E. dispar complex, whereas multiplex-PCR detected and distinguished $24.9 \%$ of their stool samples to be infected with either/or both species. They explained their findings by the higher sensitivity of multiplex PCR over microscopy in detecting Entamoeba spp. infection with $100 \%$ specificity.

In the present study, E. histolytica infection diagnosed by NM PCR proved to be higher compared with E. disparthat is, $32.7 \%$ of the examined stool samples. On the other hand, E. dispar was diagnosed in only $23 \%$. E. histolytica was more prevalent in samples collected from symptomatic cases complaining of gastrointestinal tract troubles (75\%) than in samples from the asymptomatic cases (31.2\%). The opposite occurred with E. dispar infection, which was more prevalent in asymptomatic $(56.3 \%)$ than in symptomatic patients $(12.5 \%)$. These findings oppose the results recorded by Khairnar and Parija ${ }^{[8]}$ who reported monoinfection by E. dispar in $49.5 \%$ and E. histolytica in only $7.4 \%$ of the cases examined. Herbinger et al. ${ }^{[11]}$ detected E. dispar in $88.3 \%$ and E. histolytica in $9.7 \%$ of the cases. Nohýnková et al. ${ }^{[33]}$ reported that $95.6 \%$ were positive for E. dispar, whereas only three $(4.4 \%)$ patients were positive for E. histolytica. Fallah et al. ${ }^{\left[{ }^{13]}\right.}$ reported monoinfection by E. dispar in $54.8 \%$ and E. histolytica in $25.8 \%$, and Gachuhi et al. ${ }^{[32]}$ reported monoinfection by E. dispar in $20.1 \%$ and E. histolytica in $2.4 \%$. From Menoufia governorate in Egypt, E. dispar was detected in $41.7 \%$, E. histolytica in $25 \%$, and mixed infection occurred in $33.3 \%$ of the cases analyzed by multiplex $\mathrm{PCR}^{[30]}$. In the present study, mixed infection by both species was found in $5.7 \%$ of cases, and reports of coinfection in different studies varied from $2.5^{[32]}$ to $18.8 \%{ }^{[8]}$. No coinfection was reported by Fallah et al. ${ }^{[31]}$. Compared with other reported records, the unusually high detection rate of E. histolytica in our symptomatic group may be attributed to the fact that most of the cases were from neighboring rural areas where such infections are more common.

In a study comparing the sensitivity of NM PCR with microscopy and the TechLab E. histolytica II ELISA kit, ElBakri et al. ${ }^{[25]}$ reported $19.2 \%$ (23/120) Entamoeba spp. infection by NM PCR in the United Arab Emirates.
Of those, $10 \%(12 / 120)$ included monoinfection with E. histolytica and $2.5 \%(3 / 120)$ with E. dispar. They also detected mixed infections by both $E$. histolytica and E. dispar in 3.3\% (4/120) of cases. However, the TechLab ELISA kit failed to detect E. histolytica in any of the E. histolytica PCR-positive samples, which was attributed to the low antigen levels in the fecal samples, below the detection limit of the kit, or because the assay recognizes only the amoebic adhesion of vegetative forms, normally present in diarrheal fecal specimens during an acute amoebic infection, and not the cyst-stage antigen. In addition, polymorphism in the lectin antigen used in the E. histolytica II ELISA may also explain the failure of this test. Nested PCR microscopy was found to have an overall sensitivity of $52.2 \%$ and a specificity of $75.2 \%$ for detecting the Entamoeba complex.

In conclusion, these findings demonstrate that NM PCR is a more sensitive and reliable technique that allows simultaneous, differential detection of E. histolytica and E. dispar. It is recommend that molecular techniques such as NM PCR should be used for the specific detection and differentiation of E. histolytica and E. dispar DNA in stool specimens as it is a highly specific, sensitive, rapid, and promising tool for epidemiological studies, particularly for discriminating pathogenic species from nonpathogenic species of Entamoeba.

Acknowledgement: The authors thank Dr. Ayman A. El badry, Professor of Parasitology, Kasr Al-Ainy Faculty of Medicine, Cairo University, Egypt for his kind help in performing the laboratory work.

Authors' contribution: All authors equally shared in the study design, research topics, analyzed the data, wrote and reviewed the manuscript.

Conflicts of interest: There are no conflicts of interest

\section{REFERENCES}

1. van Hal SJ, Stark DJ, Fotedar R, Marriott D, Ellis JT, Harkness JL. Amoebiasis: current status in Australia: Review. Med J Aust 2007; 186: 412-416.

2. Henrich VC. The ecdysteroid receptor. In Gill SS, Dhanalakshmi S, Parija SC. Seroprevalence of Entamoeba histolytica from a tertiary care hospital, South India. Trop Parasitol 2016; 6: 78-81.

3. Al-Harthi SA, Jamjoom M. Diagnosis and differentiation of Entamoeba infection in Makkah Al Mukarramah using microscopy and stool antigen detection kits. World J Med Sci 2007; 2: 15-20.

4. Roche J, Benito A. Prevalence of intestinal parasite infections with special reference to Entamoeba 
histolytica on the island of Bioko (Equatorial Guinea). Am J Trop Med Hyg 1999; 60: 257-262.

5. Fotedar R, Stark D, Beebe N, Marriott D, Ellis J, Harkness J. PCR detection of Entamoeba histolytica, Entamoeba dispar, and Entamoeba moshkovskii in stool samples from Sydney, Australia. J Clin Microbiol 2007; 45: 1035-1037.

6. World Health Organization. Report of the expert consultaion on amoebiasis. Weekly Epidemiological Record, No. 14, 4 April, 1997. Available at http:// Entamoeba.1shtm.ac.uk/who.htm.

7. Gomes TDS, Garcia MC, Souza Cunha FD, Werneck de Macedo H, Peralta JM, Peralta RHS. Differential diagnosis of Entamoeba spp. in clinical stool samples using SYBR green real-time polymerase chain reaction. Scientific World J 2014; 2014: 645084.

8. Khairnar K, Parija SC. A novel nested multiplex polymerase chain reaction (PCR) assay for differential detection of Entamoeba histolytica, E. moshkovskii and $E$. dispar DNA in stool samples. BMC Microbiol 2007; 7: 47.

9. Fotedar R, Stark D, Beebe N, Marriott D, Ellis J, Harkness J. Laboratory diagnostic techniques for Entamoeba species. Clin Microbiol Rev 2007; 20: 511-532.

10. Saber M, Taha AA, Mohamed SMA. Role of microscopic stool examination and copro-antigen in diagnosis of amoebic dysentery. Egypt J Med Sci 2011; 32: 633-645.

11. Herbinger $\mathrm{KH}$, Fleischmann E, Weber C, Perona P, Löscher T, Bretzel G. Epidemiological, clinical, and diagnostic data on intestinal infections with Entamoeba histolytica and Entamoeba dispar among returning travelers. Infection 2011; 39: 527-535.

12. Ngui R, Angal L, Fakhrurrazi SA, Lian YLA, Ling LY, Ibrahim J, et al. Entamoeba histolytica, Entamoeba dispar and Entamoeba moshkovskii using nested polymerase chain reaction (PCR) in rural communities in Malaysia. Parasit Vectors 2012; 5: 187.

13. Hamzah Z, Petmitr S, Mungthin M, Leelayoova S, Chavalitshewinkoon-Petmitr P. Differential detection of Entamoeba histolytica, Entamoeba dispar, and Entamoeba moshkovskii by a single-round PCR assay. J Clin Microbiol 2006; 44: 3196-3200.

14. Zebardast N, Haghighi A, Yeganeh F, Tabaei SJS, Gharavi MJ, Fallahi S et al. Application of multiplex PCR for detection and differentiation of Entamoeba histolytica, Entamoeba dispar and Entamoeba moshkovskii. Iran J Parasitol 2014; 9: 466-473.

15. Evangelopoulos A, Spanakos G, Patsoula E, Vakalis $\mathrm{N}$, Legakis N. A nested multiplex PCR assay for the simultaneous detection and differentiation of Entamoeba histolytica and Entamoeba dispar in faeces. Ann Trop Med Parasitol 2000; 94: 233-240.

16. www.who.int/iris/bitstream/10665/95584/1/ 9789241506328_eng.pdf

17. Cheesbrough M. District laboratory practice in tropical countries. New York: Cambridge University Press; 2009. p. 1.

18. Knapp R, Miller M III. Clinical epidemiology and biostatistics. Md. USA: Williams \& Wilkins; 1992. pp. 275-292.

19. Petri W, Haque R, Lyerly D, Vines R. Estimating the impact of amebiasis on health. Parasitol Today 2000; 16: $320-321$

20. Ohnishi K, Kato Y, Imamura A, Fukayama M, Tsunoda T, Sakaue Y et al. Present characteristics of symptomatic Entamoeba histolytica infection in the big cities of Japan. Epidemiol Infect 2004; 132: $57-60$

21. Nath J, Ghosh SK, Singha B, Paul J. Molecular epidemiology of amoebiasis: a cross-sectional study among North East Indian population. PLoS Negl Trop Dis 2015; 9: $\mathrm{e} 0004225$.

22. Walsh JA. Problems in recognition and diagnosis of amebiasis: estimation of the global magnitude of morbidity and mortality. Rev Infect Dis 1986; 8: $228-238$

23. Solaymani-Mohammadi S, Rezaian M, Babaei Z, Rajabpour A, Meamar AR, Pourbabai AA et al. Comparison of a stool antigen detection kit and PCR for diagnosis of Entamoeba histolytica and Entamoeba dispar infections in asymptomatic cyst passers in Iran. J Clin Microbiol 2006; 44: 2258-2261.

24. Stark D, Van Hal S, Fotedar R, Butcher A, Marriott D, Ellis J et al. Comparison of stool antigen detection kits to PCR for diagnosis of amebiasis. J Clin Microbiol 2008; 46: 1678-1681.

25. ElBakri A, Samie A, Ezzedine S, Odeh RA. Differential detection of Entamoeba histolytica, Entamoeba dispar and Entamoeba moshkovskii in fecal samples by nested PCR in the United Arab Emirates (UAE). Acta Parasitol 2013; 58: 185-190. 
26. Parija SC, Mandal J, Ponnambath DK. Laboratory methods of identification of Entamoeba histolytica and its differentiation from look-alike Entamoeba spp. Trop Parasitol 2014; 4: 90-95.

27. Parija SC. Progress in the research on diagnosis and vaccines in amebiasis. Trop Parasitol 2011; 1: 4.

28. Pinheiro SM, Carneiro RM, Aca IS, Irmao JI, Morais MA, Coimbra MR, Carvalho LB. Determination of the prevalence of Entamoeba histolytica and E. dispar in the Pernambuco state of northeastern Brazil by a polymerase chain reaction. Am J Trop Med Hyg 2004; 70: $221-224$

29. Acuna-Soto R, Samuelson J, De Girolami P, Zarate L, Millan-Velasco F, Schoolnick G, Wirth D. Application of the polymerase chain reaction to the epidemiology of pathogenic and nonpathogenic Entamoeba histolytica. Am J Trop Med Hyg 1993; 48(1): 58-70.
30. El Sobky MM, El Melegi MA, Abo-Khalil NA. Use of multiplex PCR in the differential detection of Entamoeba histolytica and/or Entamoeba dispar in comparison to microscopic examination. PUJ 2011; 4: 193-200.

31. Fallah E, Shahbazi A, Yazdanjoii M, Rahimi-Esboei B. Differential detection of Entamoeba histolytica from Entamoeba dispar by parasitological and nested multiplex polymerase chain reaction methods. J Anal Res Clin Med 2014; 2: 27-32.

32. Gachuhi S, Obonyo M, Odhiambo R, Swierczewski B, Mwakubambanya R. Differentiation of Entamoeba histolytica and Entamoeba dispar complex by multiplex polymerase chain reaction. Sch Acad J Biosci 2014; 2: 762-767.

33. Nohýnková E, Pysova I, Tůmová P, Tolarova V. Pathogenic Entamoeba histolytica-a rare incidence in persons microscopically positive for cysts in faeces. Cas Lek Cesk 2006; 146: 132-136. 